\title{
Learning Rules for Spike Timing-Dependent Plasticity Depend on Dendritic Synapse Location
}

\author{
Johannes J. Letzkus, Björn M. Kampa, and Greg J. Stuart \\ Division of Neuroscience, The John Curtin School of Medical Research, Australian National University, Canberra ACT 0200, Australia
}

Previous studies focusing on the temporal rules governing changes in synaptic strength during spike timing-dependent synaptic plasticity (STDP) have paid little attention to the fact that synaptic inputs are distributed across complex dendritic trees. During STDP, propagation of action potentials (APs) back to the site of synaptic input is thought to trigger plasticity. However, in pyramidal neurons, backpropagation of single APs is decremental, whereas high-frequency bursts lead to generation of distal dendritic calcium spikes. This raises the question whether STDP learning rules depend on synapse location and firing mode. Here, we investigate this issue at synapses between layer 2/3 and layer 5 pyramidal neurons in somatosensory cortex. We find that low-frequency pairing of single APs at positive times leads to a distance-dependent shift to long-term depression (LTD) at distal inputs. At proximal sites, this LTD could be converted to long-term potentiation (LTP) by dendritic depolarizations suprathreshold for BAC-firing or by high-frequency AP bursts. During AP bursts, we observed a progressive, distance-dependent shift in the timing requirements for induction of LTP and LTD, such that distal synapses display novel timing rules: they potentiate when inputs are activated after burst onset (negative timing) but depress when activated before burst onset (positive timing). These findings could be explained by distance-dependent differences in the underlying dendritic voltage waveforms driving NMDA receptor activation during STDP induction. Our results suggest that synapse location within the dendritic tree is a crucial determinant of STDP, and that synapses undergo plasticity according to local rather than global learning rules.

Key words: spike timing; synaptic plasticity; dendrite; backpropagating action potential; burst; calcium electrogenesis; NMDA receptor

\section{Introduction}

Synapses transfer information between neurons and have long been known to display activity-dependent, bidirectional plasticity, with a persistent increase in synaptic strength termed longterm potentiation (LTP) (Bliss and Lomo, 1973) and a persistent decrease in synaptic strength termed long-term depression (LTD) (Lynch et al., 1977). Both LTP and LTD share associativity and input specificity with certain forms of learning and memory formation, and are thus widely accepted to underlie these phenomena (for review, see Martin et al., 2000).

More recent studies show that bidirectional plasticity can be induced by the precise (millisecond) timing of presynaptic and postsynaptic action potentials (APs), in a form of plasticity termed spike timing-dependent plasticity (STDP) (for review, see Dan and Poo, 2004). When synaptic input precedes the postsynaptic AP (positive timing), LTP is typically elicited, whereas LTD is usually generated when synaptic input follows the postsynaptic AP (negative timing) (Markram et al., 1997a; Bi and Poo, 1998;

\footnotetext{
Received June 23, 2006; revised Aug. 23, 2006; accepted Aug. 25, 2006

This work was supported by the Alexander von Humboldt Foundation and The John Curtin School of Medical Research recurrent funds. J.J.L. acknowledges funding from an Australian Postgraduate Research Scholarship. We thank Stephen Williams, Maarten Kole, and Clarke Raymond for comments on this manuscript, Maarten Kole for the h-channel mod files, and Christian Stricker and Anna Cowan for help with data analysis.

Correspondence should be addressed to Greg J. Stuart at the above address. E-mail: greg.stuart@anu.edu.au.

B. M. Kampa's present address: Department Neurophysiology, Brain Research Institute, University of Zürich, 8057 Zürich, Switzerland

DOI:10.1523/JNEUROSCI.2650-06.2006

Copyright $\odot 2006$ Society for Neuroscience $\quad$ 0270-6474/06/2610420-10\$15.00/0
}

Debanne et al., 1998; Feldman, 2000; Sjostrom et al., 2001; Froemke et al., 2005). The functional interpretation of these results is that synaptic inputs that contribute to postsynaptic firing are potentiated, whereas uncorrelated inputs are depressed.

The primary feedback signal to the synapse during STDP is the AP (Magee and Johnston, 1997), which actively propagates into the dendritic tree in pyramidal neurons (Stuart and Sakmann, 1994; Spruston et al., 1995), where it presumably plays an important role in relief of the voltage-dependent block of synaptic NMDA receptors by magnesium (Mayer et al., 1984; Nowak et al., 1984).

AP backpropagation in pyramidal neurons, however, is decremental (Stuart and Sakmann, 1994; Spruston et al., 1995) and can fail at distal dendritic sites in layer 5 pyramidal neurons (Stuart and Hausser, 2001). These findings suggest that the feedback signal received by synapses during STDP will depend on their distance from the soma. In contrast to backpropagation of single APs, high-frequency AP bursts, which are a characteristic output mode of layer 5 pyramidal neurons (Connors et al., 1982), are associated with dendritic calcium electrogenesis, which is more pronounced at distal sites (Larkum et al., 1999b; Williams and Stuart, 2000b), causing qualitatively different local voltage waveforms at proximal and distal locations. This distance dependence of the feedback signal during STDP induction raises the possibility that STDP depends on synapse location (Froemke et al., 2005). To address this issue, we performed paired recordings between layer $2 / 3$ and layer 5 pyramidal neurons in brain slices. These 
experiments indicate that the timing rules for induction of STDP depend on dendritic synapse location. Our results suggest that synapses in the apical dendritic tree of layer 5 pyramidal neurons undergo plasticity according to local rather than global learning rules, and parallel previous reports of site-dependent local signal processing in these neurons (for review, see Williams and Stuart, 2003).

\section{Materials and Methods}

All animal work was performed in accordance with the guidelines approved by the Animal Ethics Committee of the Australian National University. Wistar rats (3-6 weeks of age) were anesthetized by inhalation of isoflurane and decapitated, and the brain was rapidly removed into icecold high-magnesium artificial CSF (high-Mg-aCSF) containing the following (in mM): $125 \mathrm{NaCl}, 25 \mathrm{NaHCO}_{3}, 3 \mathrm{KCl}, 1.25 \mathrm{NaH}_{2} \mathrm{PO}_{4}, 6 \mathrm{MgCl}_{2}$, and $1 \mathrm{CaCl}_{2}\left(95 \% \mathrm{O}_{2} / 5 \% \mathrm{CO}_{2}\right)$. Parasagittal slices (300 $\mu \mathrm{m}$ thick) encompassing the primary somatosensory cortex were cut on a vibrating microtome (MA752; Campden Instruments, Loughborough, UK) in icecold high-Mg-aCSF, and then transferred to aCSF containing the following (in mM): $125 \mathrm{NaCl}, 25 \mathrm{NaHCO}_{3}, 3 \mathrm{KCl}, 1.25 \mathrm{NaH}_{2} \mathrm{PO}_{4}, 1 \mathrm{MgCl}_{2}$ and $2 \mathrm{CaCl}_{2}\left(95 \% \mathrm{O}_{2} / 5 \% \mathrm{CO}_{2}\right)$ at $35^{\circ} \mathrm{C}$ for $30 \mathrm{~min}$, and allowed to cool to room temperature.

Electrophysiology. Neurons were visualized using infrared-differential interference contrast optics. Only slices in which the full apical dendritic tree of layer 5 pyramidal neurons in primary somatosensory cortex was intact were used. Whole-cell recordings were performed in aCSF $(95 \%$ $\mathrm{O}_{2} / 5 \% \mathrm{CO}_{2}$ ) at $34-35^{\circ} \mathrm{C}$ from layer 5 and layer $2 / 3$ pyramidal neurons with pipettes containing the following (in $\mathrm{mM}$ ): $100 \mathrm{~K}$-gluconate, $20 \mathrm{KCl}$, 10 HEPES, $10 \mathrm{Na}_{2}$-phosphocreatine, $4 \mathrm{Mg}$-ATP, and $0.3 \mathrm{Na}$-GTP, pH 7.4 $(\mathrm{KOH})$; osmolarity, $290 \mathrm{mOsm}$ (sucrose). Voltage, recorded in current clamp using Dagan (Minneapolis, MN) amplifiers (BVC-700A), was low-pass filtered at $10 \mathrm{kHz}$ and sampled at $20 \mathrm{kHz}$ with an ITC-18 (Instrutech, Port Washington, NY) analog-to-digital converter using Axograph (Molecular Devices, Union City, CA) software running on a Macintosh computer. Recordings were only included if the series resistance was $<20 \mathrm{M} \Omega$ for layer 5 somata, $<30 \mathrm{M} \Omega$ for layer $2 / 3$ somata, and $<50 \mathrm{M} \Omega$ for layer 5 dendrites. Series resistance was compensated using the bridge balance and capacitance neutralization facilities of the amplifier. The liquid junction potential was left uncorrected. Membrane potential was $-66.3 \pm 1.2 \mathrm{mV}$ in layer 5 and $-81.0 \pm 3.4 \mathrm{mV}$ in layer $2 / 3$ pyramidal neurons (mean \pm SD) and remained within $10 \%$ of the initial value during the course of an experiment. Input resistance was $20.9 \pm 3.9$ $\mathrm{M} \Omega$ in layer 5 and $39.7 \pm 10.5 \mathrm{M} \Omega$ in layer $2 / 3$ pyramidal neurons (mean \pm SD) and did not change $>20 \%$ during recordings. Drugs were bath applied: $50 \mu \mathrm{M}$ 4-ethylphenylamino-1,2-dimethyl-6-methylaminopyrimidinium chloride (ZD7288) (Tocris, Ellisville, MO), $50 \mu \mathrm{M}$ D-APV (Tocris), and $100 \mu \mathrm{M} \mathrm{NiCl}$ (Merck). APV and $\mathrm{NiCl}_{2}$ were washed in before STDP induction and washed out immediately after induction.

Paired recording and synaptic plasticity. Paired somatic recordings from layer 5 and layer $2 / 3$ pyramidal neurons were established and connectivity was tested by generating $>20$ APs in the layer $2 / 3$ neuron. Unitary EPSPs (uEPSPs) were evoked at $0.25 \mathrm{~Hz}$. A baseline period of 10 min was recorded before induction. Experiments in which the uEPSP amplitude changed $>20 \%$ during baseline were discarded. Plasticity was induced by pairing presynaptic and postsynaptic APs 100-200 times at 1 $\mathrm{Hz}$, after which the pair was routinely recorded for $30 \mathrm{~min}$ to assess plasticity. When tested, changes in uEPSP amplitude remained for the duration of recordings (up to $60 \mathrm{~min}$ after induction; LTP, $n=4$; LTD, $n=6$ ) (data not shown). All APs were elicited by brief somatic current injections (2 ms; 3-5 nA). Plasticity was defined as the average uEPSP amplitude 20-30 min after induction divided by the average amplitude during baseline. Identical experiments without postsynaptic activity (plasticity, $0.98 \pm 0.06 ; p>0.05 ; n=6$ ) or with postsynaptic AP bursts offset by $500 \mathrm{~ms}$ during STDP induction (plasticity, $0.99 \pm 0.05 ; p>$ $0.05 ; n=5$ ) did not cause significant changes to the uEPSP amplitude. EPSP rise time was calculated from 10 to $90 \%$ of peak amplitude. uEPSPs with rising phases consisting of more than one component were rare ( $\sim 1 \%$ of connections) and were not included in the analysis.
Imaging. To visualize synaptic contacts made by layer $2 / 3$ onto layer 5 pyramidal neurons, pairs were identified as described above, and then repatched with internal solution containing Alexa 594 (Invitrogen, Eugene, OR) (layer 5 neuron, $50 \mu \mathrm{M}$; layer $2 / 3$ neuron, $200 \mu \mathrm{M}$ ). After filling cells for $>1 \mathrm{~h}$, they were visualized using a two-photon laser scanning microscope (LSM 510; Zeiss, Oberkochen, Germany). This made it possible to follow the main axon and collaterals of layer $2 / 3$ neurons reliably. Putative synaptic contacts were defined as close appositions $(<2 \mu \mathrm{m})$ between the presynaptic layer $2 / 3$ axon and a postsynaptic layer 5 dendrite.

Dendritic recording. Paired recordings from the soma and the apical dendrite of layer 5 pyramidal neurons were performed as described (Stuart and Sakmann, 1994). EPSPs were evoked by a bipolar stimulating electrode placed in close proximity to the dendritic recording site using brief current pulses (100 $\mu \mathrm{s} ; 50-200 \mu \mathrm{A})$. Artificial EPSPs (aEPSPs) were generated by current injection $\left(\tau_{\text {rise }}, 0.2 \mathrm{~ms} ; \tau_{\text {decay }}, 2 \mathrm{~ms} ; 600 \mathrm{pA}\right)$ (Hausser and Roth, 1997) via the dendritic or a second somatic recording pipette and recorded at the soma.

Statistics. Data are reported as mean \pm SEM unless stated otherwise. All data were subjected to a Kolmogorov-Smirnov normality test before additional analysis. Statistical significance of the effect of pairing paradigms on uEPSP amplitude (including EPSP alone) was calculated using a two-tailed, paired $t$ test of uEPSP amplitude during baseline versus 20-30 min postinduction. Statistical significance of APV and $\mathrm{NiCl}_{2}$ applications was determined using a two-tailed, unpaired $t$ test of plasticity values in control versus drug. Correlations were calculated using the Pearson test. The significance level was 0.05 .

Modeling. Induction of STDP was simulated with NEURON 5.7 (Hines and Carnevale, 1997) using a reconstructed layer 5 pyramidal neuron model (Stuart and Spruston, 1998). The specific membrane resistance was set to $15 \mathrm{k} \Omega \cdot \mathrm{cm}^{2}$, the membrane capacitance was $1 \mu \mathrm{F} /$ $\mathrm{cm}^{2}$, the axial resistance was $125 \Omega \cdot \mathrm{cm}$. Spines were incorporated by decreasing the membrane resistance and increasing the membrane capacitance twofold in spiny compartments. To account for active properties, voltage-gated channels (Schaefer et al., 2003) were incorporated at the following densities: The soma contained (in $\mathrm{pS} / \mu \mathrm{m}^{2}$ ) $g_{\mathrm{Na}}=3000$, $g_{\mathrm{Kv}}=30, g_{\mathrm{Ka}}=0.06, g_{\mathrm{Kca}}=2.5, g_{\mathrm{Km}}=2.2$, and T-type calcium conductance $g_{\mathrm{CaT}}=0.0003$. In the dendrites, the channel densities were as follows: $g_{\mathrm{Na}}=40, g_{\mathrm{Kv}}=30, g_{\mathrm{Ka}}=0.03, g_{\mathrm{Kca}}=2.5, g_{\mathrm{Km}}=0.05$, and $g_{\mathrm{CaT}}=0.0003$. In distal dendrites $\left(>600 \mu \mathrm{m}\right.$ from soma), $g_{\mathrm{CaT}}$ was 0.001 and a slow high-voltage activated calcium conductance was inserted with $g_{\mathrm{Ca}}=1.25$. In the reconstructed axon, $g_{\mathrm{Na}}$ was 30,000 and $g_{\mathrm{Kv}}$ was 400 . Additionally, $I_{\mathrm{h}}$ was inserted according to a recent study (Kole et al., 2006). We set the somatic resting membrane potential to $-80 \mathrm{mV}$ and AP threshold to $-63 \mathrm{mV}$ (Gulledge and Stuart, 2003). The nominal temperature was set to $35^{\circ} \mathrm{C}$. A synapse was inserted at the specified dendritic location and contained an AMPA component $\left(0.2 \mathrm{nA} ; \tau_{\text {rise }}=\right.$ $0.2 \mathrm{~ms} ; \tau_{\text {decay }}=2 \mathrm{~ms}$ ) and an NMDA component that was scaled to achieve an NMDA-AMPA ratio of 0.2 (Watt et al., 2004). The NMDA conductance was simulated with a realistic kinetic model as described by Kampa et al. (2004). For every dendritic location, the synaptic response was paired with a burst of APs at different spike timing intervals. STDP was calculated using a threshold model of synaptic plasticity induction (Shouval et al., 2002) based on the magnitude of NMDA receptor activation. At each synapse location integrated NMDA conductance was normalized to the maximum observed during STDP. Above 55\% NMDA activation, the magnitude of plasticity was calculated using the following function: STDP $=2.15-1.65 \times \exp \left(-((\mathrm{NMDA}-0.64) / 0.23)^{2}\right)($ supplemental Fig. 5, available at www.jneurosci.org as supplemental material).

This led to LTD between 55 and $80 \%$, and LTP $>80 \%$, of maximum NMDA activation. In addition, the maximal and minimum values of LTP and LTD were set to 200 and $50 \%$ of baseline, respectively. These parameters were chosen to best fit the experimental observations. The spike timing interval for maximum LTP induction (see Fig. $8 \mathrm{C}$ ) was calculated from the peaks of Gaussian fits to STDP timing curves. Modeling data were analyzed in IGOR (Wavemetrics, Lake Oswego, OR). 
A

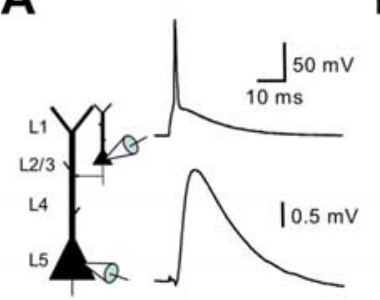

B

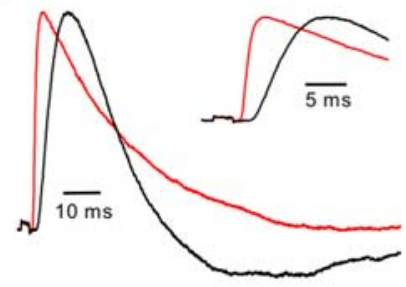

C

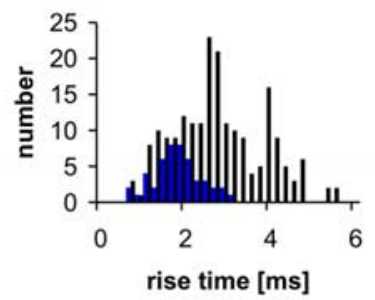

D
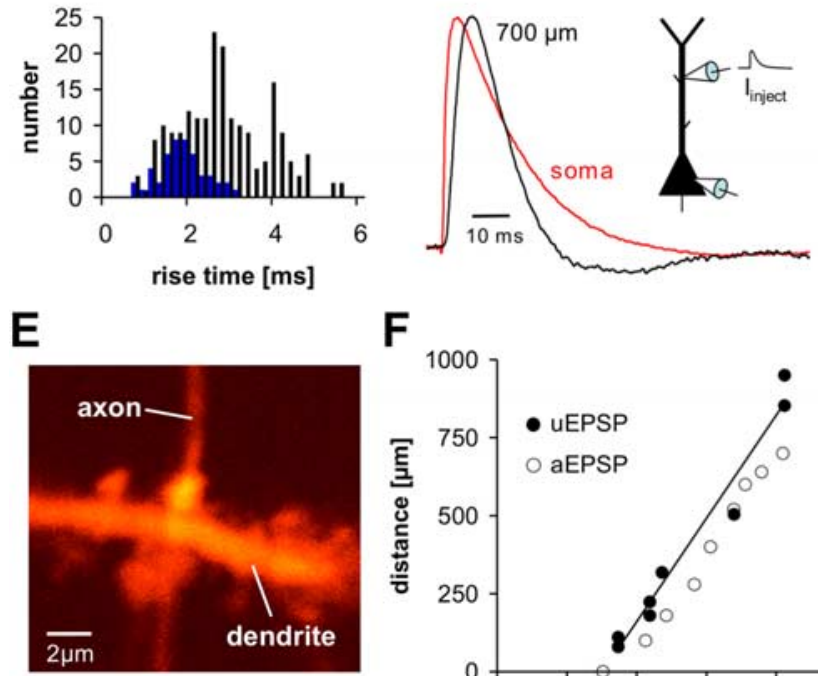

$F$

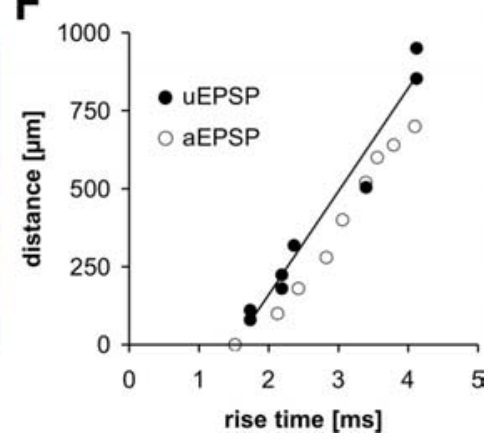

Figure 1. Layer $2 / 3$ inputs are distributed throughout the apical dendrites of layer 5 pyramidal neurons. A, Experimental setup for paired somatic whole-cell current-clamp recordings between synaptically connected layer $2 / 3$ and layer 5 pyramidal neurons. A presynaptic AP in the layer $2 / 3$ neuron (top trace) elicits a unitary EPSP in the layer 5 neuron (uEPSP; bottom trace; average of 50 sweeps). $\boldsymbol{B}$, uEPSPs display different rise times. Time course of a fast rising (red) and a slow rising (black) uEPSP (normalized averages of 100 sweeps). Note that only the slow uEPSP (black) shows an afterhyperpolarization, consistent with activation of $I_{\mathrm{h}}$ in the distal dendrites. C, Layer 2/3 uEPSPs show a broad distribution of EPSP rise times (black; $n=191$ ) with a median of $2.7 \mathrm{~ms}$, and are significantly slower than layer 5 to layer 5 uEPSPs (blue; median, $1.6 \mathrm{~ms} ; p<0.001 ; n=48)$. $D$, Setup for dual recordings from the soma and apical dendrite of layer 5 pyramidal neurons (inset). Artificial EPSCS (aEPSCS) were injected $\left(I_{\text {inject }}\right.$ ) at a distal apical location $(700 \mu \mathrm{m})$ or at the soma, and recorded via a second pipette at the soma. Normalized aEPSPs display a fast rise time when generated by somatic current injection (red; average of 25 sweeps), whereas aEPSPs generated by distal dendritic current injection rise more slowly (black; $700 \mu \mathrm{m}$; average of 25 sweeps). $\boldsymbol{E}$, Collapsed two-photon $z$-stack (0.9 $\mu \mathrm{m}$ thickness) of a putative synaptic contact between a layer $2 / 3$ axon and a layer 5 dendrite both filled with Alexa 594. $\boldsymbol{F}$, The physical distance of putative synaptic contacts from the soma of layer 5 pyramidal neurons correlates linearly with uEPSP rise time (closed circles with linear fit; $r=$ $0.98 ; p<0.001 ; 5$ pairs with 8 contacts). aEPSPs injected into sites on the apical dendrite display a similar correlation (open circles).

\section{Results}

EPSP rise time as an indicator of synaptic location

To address the potential role of synapse location in STDP, we made paired whole-cell recordings from synaptically connected layer $2 / 3$ and layer 5 pyramidal neurons in brain slices of somatosensory cortex from 3 - to 6 -week-old rats (Fig. $1 A$ ). The average probability of finding a connected pair was $0.17(n>500)$ for layer $2 / 3$ neurons in the immediate vicinity of the layer 5 apical dendritic tree. uEPSPs generated by APs in layer $2 / 3$ pyramidal neurons and recorded at the soma of layer 5 pyramidal neurons displayed a range of rise times (Fig. $1 B)(0.7-5.5 \mathrm{~ms} ; n=191)$, with a median of $2.7 \mathrm{~ms}$ (Fig. $1 C$, black). This was significantly slower than the rise time of uEPSPs recorded in layer 5 to layer 5 pairs (Fig. 1C, blue). Previous theoretical work indicates that, in a passive system, the rise time of somatic EPSPs is dependent on the electrotonic distance of the synapse from the soma (Jack et al., 1971). Consistent with this idea, aEPSPs generated by current injection at different distances from the soma of layer 5 pyramidal neurons displayed a similar range of somatic EPSP rise times (Fig. $1 D, F)(1.5-4.1 \mathrm{~ms})$. To directly identify the location of synapses between layer $2 / 3$ and layer 5 pyramidal neurons, synaptically coupled pairs were filled with a fluorescent dye (Alexa 594). $\mathrm{Pu}-$ tative contacts were visualized using two-photon laser-scanning microscopy by following the presynaptic layer $2 / 3$ axon and collaterals to points of close apposition with a layer 5 dendrite (Fig. $1 E)$. Contacts were found on proximal oblique dendrites, the main apical trunk and secondary dendrites in the apical tuft, consistent with previous work (Reyes and Sakmann, 1999). In cases where two putative contacts were found, these were always at comparable distances from the layer 5 soma, suggesting that, under our recording conditions, layer $2 / 3$ inputs make relatively localized inputs onto layer 5 dendrites. Importantly, somatic $\mathrm{uEPSP}$ rise time correlated with the physical distance of putative layer 2/3 contacts from the layer 5 soma (Fig. $1 F$, closed circles with linear fit $)(r=0.98 ; p<0.001)$. A similar correlation was observed between somatic aEPSP rise time and the distance of dendritic current injection from the soma (Fig. $1 F$, open circles) $(r=0.99 ; p<0.001)$, and in computer simulations (supplemental Fig. 1, available at www.jneurosci.org as supplemental material). These results suggest that the range of observed uEPSP rise times is attributable to different extents of filtering by the apical dendrite rather than differences in synaptic physiology, and that slower uEPSP rise times are associated with more distal synaptic inputs, consistent with previous results (Williams and Stuart, 2002). Additional evidence that the synaptic contacts generating unitary layer 2/3 EPSPs are made primarily onto the apical dendritic tree of layer 5 neurons comes from the finding that uEPSPs recorded by a dendritic recording pipette on the apical trunk (429 $\pm 13 \mu \mathrm{m}$ from the soma) displayed significantly faster rise times $(p<0.001 ; n=16)$ and larger amplitudes $(p<0.01 ; n=$ 16) than at the soma (supplemental Fig. 2, available at www. jneurosci.org as supplemental material). Finally, correlations of synapse location, as well as the site of dendritic current injection, with physical distance from the soma indicate that uEPSPs from layer $2 / 3$ neurons make synaptic connections over a large region of the apical dendrite of layer 5 pyramidal neurons (Fig. $1 F$ ).

Although these data are consistent with passive filtering of uEPSP time course, previous work indicates that layer 5 pyramidal neuron dendrites contain voltage-gated channels, most notably $I_{\mathrm{h}}$, which has been shown to influence EPSP time course in a distance-dependent manner (Williams and Stuart, 2000a). Consistent with this idea, uEPSP rise time and half-width were not correlated under control conditions (supplemental Fig. $3 A$, available at www.jneurosci.org as supplemental material). A clear correlation between uEPSP rise time and half-width was observed, however, after block of $I_{\mathrm{h}}$ by application of ZD7288 (supplemental Fig. $3 B$, available at www.jneurosci.org as supplemental material), as expected for a passive system. uEPSP rise time in control and ZD7288 were highly correlated (supplemental Fig. 3C, available at www.jneurosci.org as supplemental material), indicating that, despite the presence of voltage-activated channels in the apical dendrites of layer 5 pyramidal neurons, somatic uEPSP rise time under control conditions can be used as a measure of synaptic distance from the soma. In conclusion, we show that layer 2/3 pyramidal neurons make synaptic inputs over a large region 

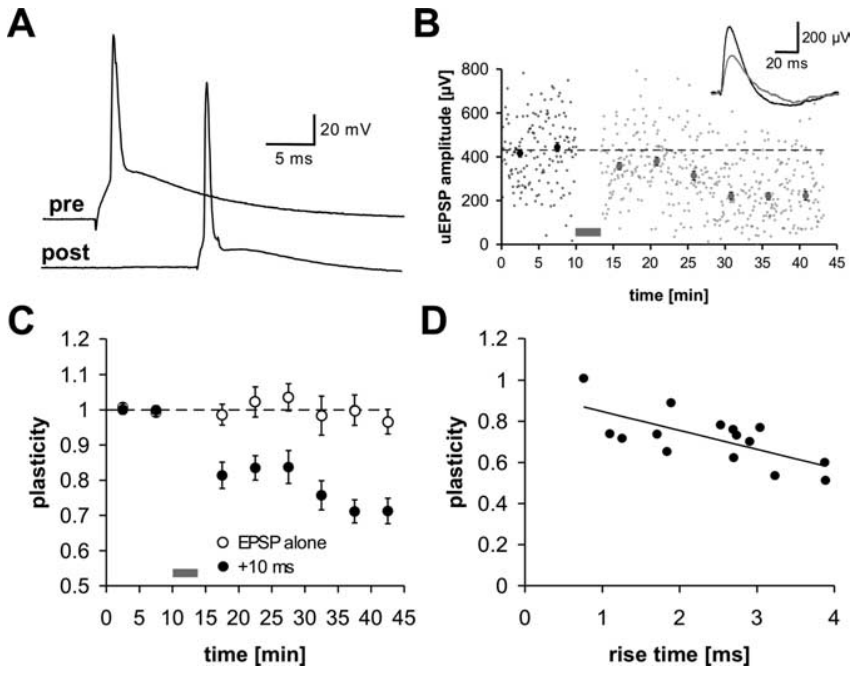

Figure 2. Pairing single APs at +10 ms leads to distance-dependent LTD. $A$, Paradigm used to induce STDP. The presynaptic AP was evoked $10 \mathrm{~ms}$ before the postsynaptic AP. B, Time course of one experiment. uEPSPs were evoked for a $10 \mathrm{~min}$ baseline period (small black circles, single trials; larger black circles, 5 min averages). After pairing (gray bar), uEPSP amplitude displayed a persistent reduction (small gray circles, single trials; larger gray circles, 5 min averages). The line represents the average uEPSP amplitude during baseline. Inset are the average uEPSPs during baseline (black; 150 sweeps) and $20-30$ min after pairing (gray; 150 sweeps). $C$ The average time course of LTD from all single AP pairing experiments (closed circles; 5 min averages; $n=15$ ) shows that LTD onset is immediate and that it develops further until reaching steady state $20-30 \mathrm{~min}$ after induction. uEPSPs elicited without postsynaptic activity $(n=6)$ or with postsynaptic AP bursts offset by $500 \mathrm{~ms}(n=5)$ showed no change in amplitude (open circles, pooled data; $p>0.05$ ). $D$, The magnitude of LTD correlates with uEPSP rise time. Plasticity was defined as the average uEPSP amplitude $20-30 \mathrm{~min}$ after induction divided by the average baseline amplitude. Plasticity correlates with synapse location over the range of rise times examined $(r=-0.69 ; p<0.01 ; n=15)$. Data are presented as mean \pm SEM.

of the apical dendrite of layer 5 pyramidal neurons, and that their physical distance from the soma can be inferred from the somatic uEPSP rise time.

Single AP pairing at $+\mathbf{1 0} \mathbf{m s}$ causes distance-dependent LTD We first addressed the distance dependence of STDP by investigating plasticity at layer $2 / 3$ to layer 5 synapses during pairing of single presynaptic and postsynaptic APs at low frequencies (1 $\mathrm{Hz}$ ). Previous studies indicate that positive spike timing typically leads to LTP (for review, see Dan and Poo, 2004). In contrast, pairing presynaptic and postsynaptic APs at $+10 \mathrm{~ms}$ (Fig. $2 \mathrm{~A}$ ) elicited LTD (Fig. $2 B, C$ ). In our experiments, LTD was apparent immediately after induction, and developed further until reaching equilibrium $\sim 20 \mathrm{~min}$ postinduction (Fig. $2 C$ ). This time course is comparable with previous studies of neocortical LTD (Egger et al., 1999; Feldman, 2000; Sjostrom et al., 2001). Experiments were routinely conducted until $30 \mathrm{~min}$ postinduction, and plasticity was defined as the average uEPSP amplitude 20-30 min after induction divided by the average $10 \mathrm{~min}$ before induction. Layer $2 / 3 \mathrm{uEPSPs}$ were on average depressed to $0.72 \pm 0.03$ $(n=15 ; p<0.001)$ of control after pairing with single postsynaptic APs in layer 5 neurons at a time interval of $+10 \mathrm{~ms}$ (pre before post). LTD was associative because uEPSPs that were not paired with postsynaptic APs, or paired with APs offset by 500 ms, showed no change (Fig. $2 C$ ) (plasticity, $0.98 \pm 0.04 ; p>0.05$; $n=11)$.

To investigate the dependence of the observed LTD on synapse location, we plotted the extent of plasticity versus somatic uEPSP rise time (Fig. $2 D$ ). Analysis of the data in this way indi- cated that the magnitude of LTD was correlated with uEPSP rise time $(r=-0.69 ; p<0.01 ; n=15)$ and that distal layer $2 / 3$ connections depressed to a greater extent than proximal connections (Fig. 2D). These experiments indicate that, for the same presynaptic and postsynaptic partners, STDP learning rules depend on synapse location. Furthermore, they suggest that, under the conditions applied here, the depolarization associated with single backpropagating APs is insufficient to generate LTP.

\section{Impact of dendritic depolarization on STDP induction}

The propagation of APs into the apical dendrite of layer 5 pyramidal neurons is modulated by dendritic depolarization (Larkum et al., 2001; Stuart and Hausser, 2001), which can lead to a phenomenon called BAC-firing (Larkum et al., 1999a), in which single APs paired with dendritic depolarization generate dendritic calcium spikes and subsequent AP burst firing. These findings suggest that dendritic depolarization may influence the induction of STDP. Furthermore, in vivo studies indicate that the entire apical dendritic tree of cortical pyramidal neurons can be depolarized during up states (Waters and Helmchen, 2004). We therefore investigated the impact of dendritic depolarization on STDP. Apical dendrites were depolarized via dendritic current injection ( $100 \mathrm{~ms} ; 429 \pm 14 \mu \mathrm{m}$ from the soma) during pairing of presynaptic and postsynaptic APs at $+10 \mathrm{~ms}$ (Fig. 3). Dendritic current injections were either subthreshold (Fig. $3 A$, left) or suprathreshold (Fig. $3 A$, right) for the generation of AP bursts via BAC-firing (Larkum et al., 1999a). These experiments showed that dendritic depolarizations $(12.1 \pm 0.9 \mathrm{mV} ; n=6)$ that were subthreshold for BAC-firing lead to LTD (Fig. 3B, left), as observed in the absence of dendritic current injection (Fig. $2 B, C$ ). Furthermore, the distance dependence of LTD during pairing of presynaptic and postsynaptic APs at $+10 \mathrm{~ms}$ (Fig. 2D) was unaffected by dendritic depolarization subthreshold for BAC-firing (Fig. 3C, open vs gray circles).

In contrast, during more powerful dendritic depolarization $(24.1 \pm 2.5 \mathrm{mV})$ leading to BAC-firing, pairing at $+10 \mathrm{~ms}$ elicited LTP at proximal synapses (Fig. $3 B$, right). Plotting plasticity in these experiments against uEPSP rise time revealed a strong negative correlation (Fig. $3 C$, black circles) $(r=-0.95$; $p<0.001$; $n=7)$. We conclude from these experiments that dendritic calcium electrogenesis and/or associated somatic burst firing are required for LTP induction at low pairing frequencies $(1 \mathrm{~Hz})$. Furthermore, these experiments show that up state like dendritic depolarizations subthreshold for BAC-firing are not sufficient to convert LTD into LTP during pairing of single presynaptic and postsynaptic APs at low frequencies.

\section{During AP bursts, the timing requirements for LTP and LTD differ at proximal and distal synapses}

To further explore the role of AP burst firing and associated dendritic electrogenesis in STDP, we evoked AP bursts via somatic current injection. AP burst firing in layer 5 pyramidal neurons (typically at $200-300 \mathrm{~Hz}$ ) has been described in vitro (Connors et al., 1982), as well as in vivo (Llinas, 1988; Manns et al., 2004), and is a distinct output mode of these neurons. Previous work indicates that high-frequency $(>100 \mathrm{~Hz})$ AP bursts generate substantial dendritic depolarization because of activation of both dendritic sodium and calcium channels (Larkum et al., 1999b; Williams and Stuart, 1999). We investigated the impact of pairing unitary layer 2/3 EPSPs generated at different dendritic locations with postsynaptic AP bursts at different timings (Fig. $4 A, E$ ). Stereotyped AP bursts (3 APs at $200 \mathrm{~Hz}$ ) were elicited in 
A

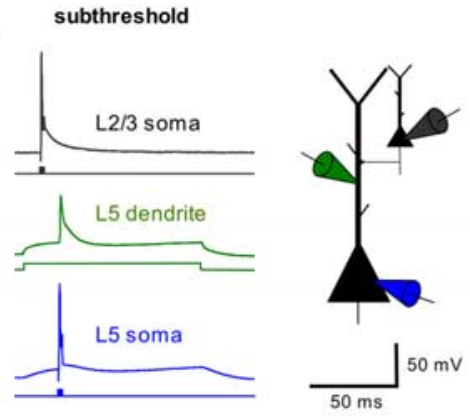

BAC-firing

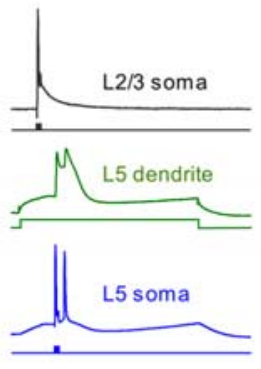

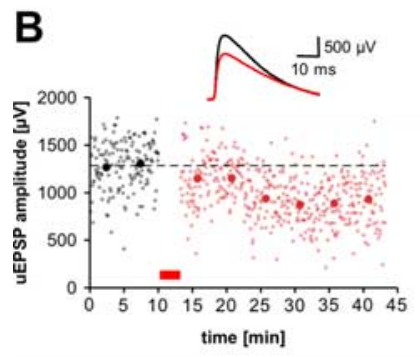

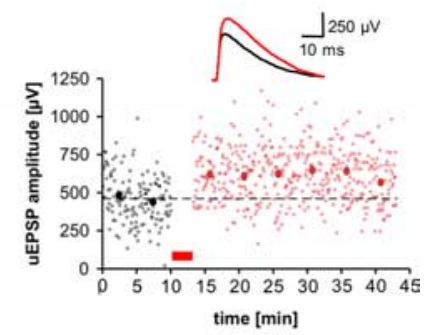

C

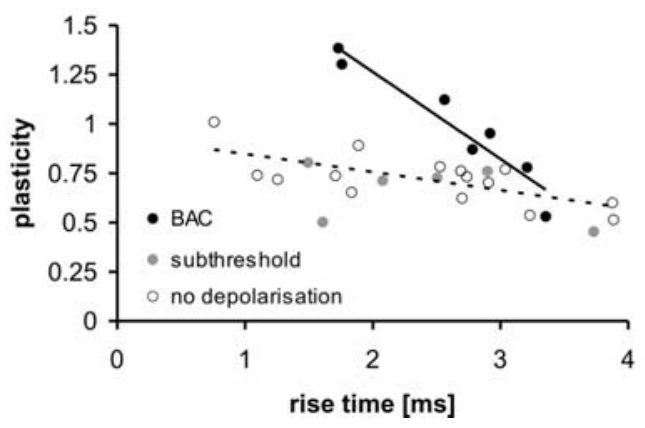

Figure 3. Effect of dendritic depolarization on STDP induction. $\boldsymbol{A}$, The experimental setup (center) consisting of a paired somatic recording between synaptically connected layer 2/3 (gray pipette) and layer 5 (blue pipette) pyramidal neurons combined with a dendritic layer 5 recording (green pipette; $429 \pm 14 \mu \mathrm{m}$ from the soma). During plasticity induction, a single presynaptic and postsynaptic AP was elicited at $+10 \mathrm{~ms}$ and paired with dendritic depolarization (100 ms), which was either just subthreshold (left) or suprathreshold (right) for BAC-firing. $B$, Examples of experiments over time (small symbols, single trials; large symbols, 5 min averages; pairing indicated by red bar; line represents average baseline uEPSP amplitude). Left, Subthreshold dendritic depolarization caused LTD. Right, Suprathreshold dendritic depolarization leading to BAC-firing caused LTP. C, Summary plot of plasticity against uEPSP rise time for three sets of experiments. Open circles, Pairing of single presynaptic and postsynaptic APs at +10 ms without dendritic depolarization (same data as in Fig. 2D). Gray circles, Experiments with subthreshold postsynaptic depolarization. Black circles, BAC-firing experiments. Note that recordings with or without subthreshold dendritic depolarization yield similar results, whereas BAC-firing at +10 ms elicits LTP at proximal connections, but LTD at distal connections, leading to a negative correlation between plasticity and uEPSP rise time ( $r=$ $-0.96 ; p<0.001 ; n=7)$.

layer 5 pyramidal neurons by three brief somatic current injections, with their timing defined by the first $\mathrm{AP}$ in the burst.

Low-frequency pairing $(1 \mathrm{~Hz})$ of unitary layer 2/3 EPSPs with postsynaptic AP bursts with a pre-before-post timing of $+10 \mathrm{~ms}$ (Fig. 4A) elicited different effects at proximal and distal synapses, with proximal uEPSPs displaying LTP and distal uEPSPs showing LTD (Fig. $4 B, C$ ). This led to a clear negative correlation between uEPSP rise time and plasticity over the whole range of synaptic locations examined (Fig. 4D) $(r=-0.69 ; p<0.001 ; n=20)$. This result is consistent with our observations during dendritic current injection suprathreshold for BAC-firing (Fig. 3C). Together, these data indicate that during low-frequency pairing

A

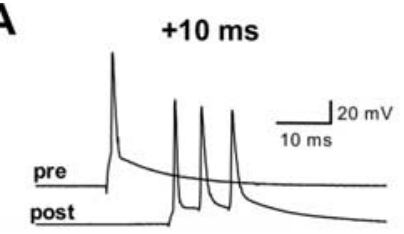

B

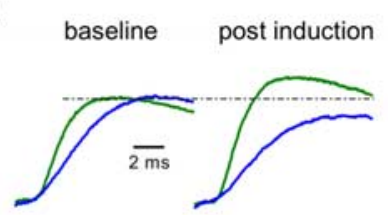

C

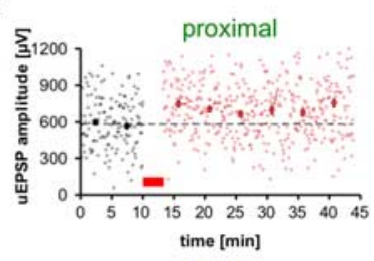

E

$-10 \mathrm{~ms}$

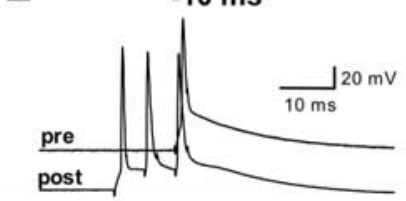

$\mathbf{F}$

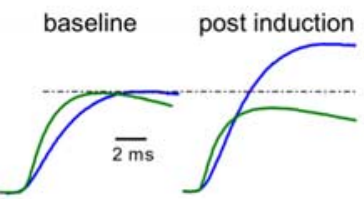

G

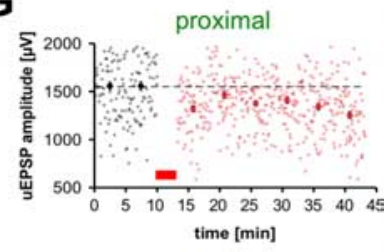

distal

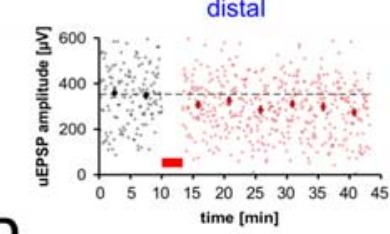

D
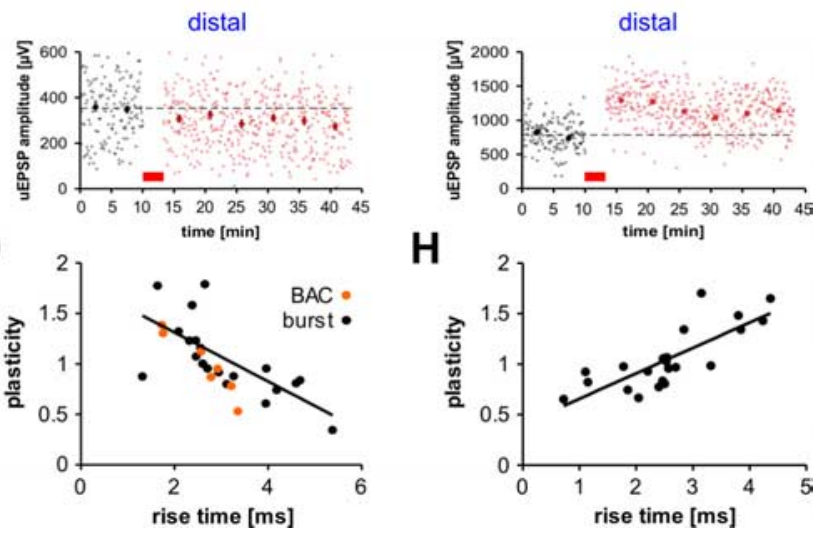

H

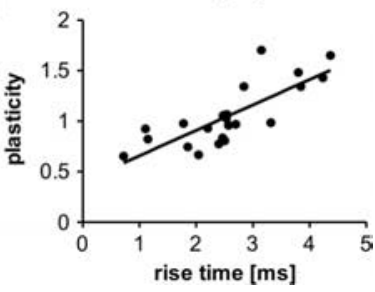

Figure 4. During AP bursts, the timing requirements for STDP differ at proximal and distal synapses. $A$, Paradigm used to induce STDP during pairing of presynaptic APs $10 \mathrm{~ms}$ before the onset of the postsynaptic AP burst ( $+10 \mathrm{~ms} ; 3$ action potentials at $200 \mathrm{~Hz}$ ). $\boldsymbol{B}$, Examples of plasticity for uEPSPs with different rise times (averages of 150 sweeps each; normalized to baseline; dotted line). Pairing at $+10 \mathrm{~ms}$ causes potentiation of the fast rising and depression of the slow rising uEPSP. C, Example experiments from $\boldsymbol{B}$ over time (small symbols, single trials; large symbols, 5 min averages; pairing indicated by red bar; line represents average baseline uEPSP amplitude). $\boldsymbol{D}$, Plot of the plasticity outcome in response to pairing AP bursts (black) at $+10 \mathrm{~ms}$ against uEPSP rise time reveals a negative correlation over the whole range of rise times tested $(r=-0.69 ; p<0.001 ; n=20)$. Note overlap with data for AP bursts evoked by BAC-firing (from Fig. 3C; orange). E, Paradigm used to induce STDP during pairing of presynaptic APs $10 \mathrm{~ms}$ after the onset of the postsynaptic AP burst $(-10 \mathrm{~ms})$. $\boldsymbol{F}$, Examples of plasticity for uEPSPs with different rise times (averages of 150 sweeps each; normalized to baseline; dotted line). Pairing at $-10 \mathrm{~ms}$ causes depression of the fast-rising and potentiation of the slow-rising uEPSPs. G, Example experiments from $\boldsymbol{F}$ over time (small symbols, single trials; large symbols, 5 min averages; pairing indicated by red bar; line represents average baseline uEPSP amplitude). $\boldsymbol{H}$, Plot of the plasticity outcome attributable to pairing at $-10 \mathrm{~ms}$ indicates a positive correlation between plasticity and uEPSP rise time $(r=0.79 ; p<0.001 ; n=23)$.

with postsynaptic AP bursts at +10 ms proximal inputs undergo LTP, whereas distal layer 2/3 inputs undergo LTD.

This result could be attributable to the distance dependence of the postsynaptic depolarization during AP bursts (see below) or to differences in the physiology of proximal and distal synapses, such as the paired-pulse ratio (Williams and Stuart, 2002), which could in principle bias proximal inputs toward LTP and distal inputs toward LTD. To distinguish between these two possibilities, we performed low-frequency pairing with postsynaptic AP bursts at a post-before-pre timing of $-10 \mathrm{~ms}$ (Fig. $4 E$ ). Using this pairing paradigm, we observed the opposite result from $+10 \mathrm{~ms}$ 


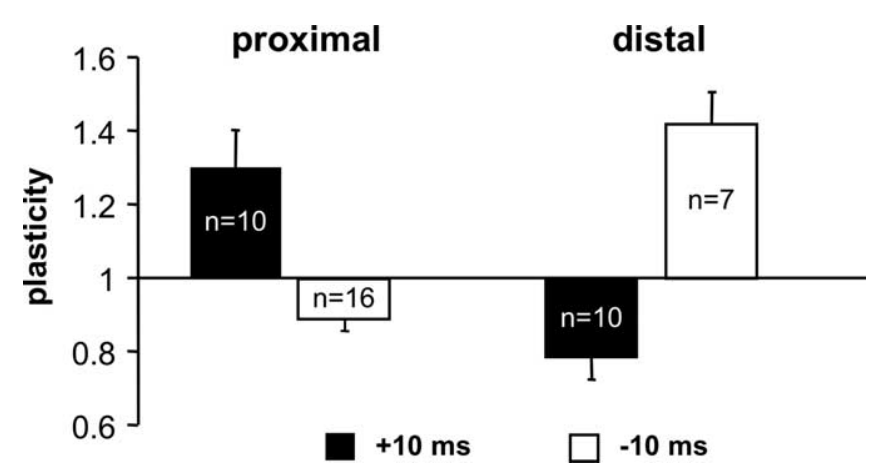

Figure 5. Summary of plasticity induced by AP bursts. uEPSPs were classified as proximal or distal based on their rise time (proximal: rise time, $<2.7 \mathrm{~ms}$; distal: rise time, $>2.7 \mathrm{~ms}$ ). Proximal uEPSPs potentiate during pairing at +10 ms with postsynaptic AP bursts $(p<0.05)$ but depress after pairing at $-10 \mathrm{~ms}(p<0.05)$. Conversely, distal uEPSPs depress during pairing at $+10 \mathrm{~ms}(p<0.001)$ but potentiate during pairing at $-10 \mathrm{~ms}(p<0.05)$. Data are presented as mean \pm SEM.

pairing, with proximal layer $2 / 3$ uEPSPs showing LTD, and distal uEPSPs undergoing LTP (Fig. $4 F, G$ ). In contrast to the distance dependence of plasticity during pairing at $+10 \mathrm{~ms}$, pairing uEPSP with AP bursts at $-10 \mathrm{~ms}$ led to a clear positive correlation between uEPSP rise time and plasticity (Fig. $4 H)(r=0.79$; $p<$ $0.001 ; n=23)$. We conclude that differences in synapse properties are unlikely to underlie the observed distance dependence of STDP, because changing the timing during induction from +10 to $-10 \mathrm{~ms}$ caused opposite correlations of plasticity and synapse location.

Based on a median uEPSP rise time of $2.7 \mathrm{~ms}$ (Fig. 1C), we classified layer $2 / 3 \mathrm{uEPSPs}$ as proximal if the uEPSP rise time was $<2.7 \mathrm{~ms}$ or distal if the uEPSP rise time was $>2.7 \mathrm{~ms}$. On average, pairing uEPSPs with postsynaptic AP bursts at $+10 \mathrm{~ms}$ led to a potentiation of proximal inputs to $1.3 \pm 0.1(p<0.05 ; n=10)$ of control, and depression of distal inputs to $0.79 \pm 0.06(p<$ $0.001 ; n=10$ ). In contrast, pairing uEPSPs with postsynaptic AP bursts at $-10 \mathrm{~ms}$ led to a small, but significant, depression of proximal inputs to $0.89 \pm 0.03(p<0.05 ; n=16)$ of control, whereas distal inputs were potentiated to $1.42 \pm 0.09(p<0.05$; $n=7$ ). These results are summarized in Figure 5 and show that, during pairing of uEPSPs with AP bursts, the timing requirements for LTP and LTD induction depend on synapse location. In addition, distal inputs show novel timing requirements because they depress at positive and potentiate at negative timings.

\section{AP bursts generate a distance-dependent increase in dendritic calcium electrogenesis}

What cellular mechanisms underlie the dependence of STDP on synapse location? As mentioned above, single APs attenuate as they propagate into the apical dendrites of layer 5 pyramidal neurons, whereas AP bursts lead to distal dendritic calcium electrogenesis. These data indicate that the local voltage during single APs and AP bursts will vary with dendritic location. To determine the voltage waveform experienced by synapses at different dendritic locations during STDP induction we performed simultaneous recordings from the soma and the apical dendrite of layer 5 pyramidal neurons. In addition, an extracellular stimulating electrode was placed in close proximity $(<30 \mu \mathrm{m})$ to the dendritic recording site to elicit EPSPs. EPSP rise time was in all cases significantly faster at the dendritic compared with somatic recording site, indicating that EPSPs were indeed generated close to the dendritic recording pipette. At proximal dendritic sites, the
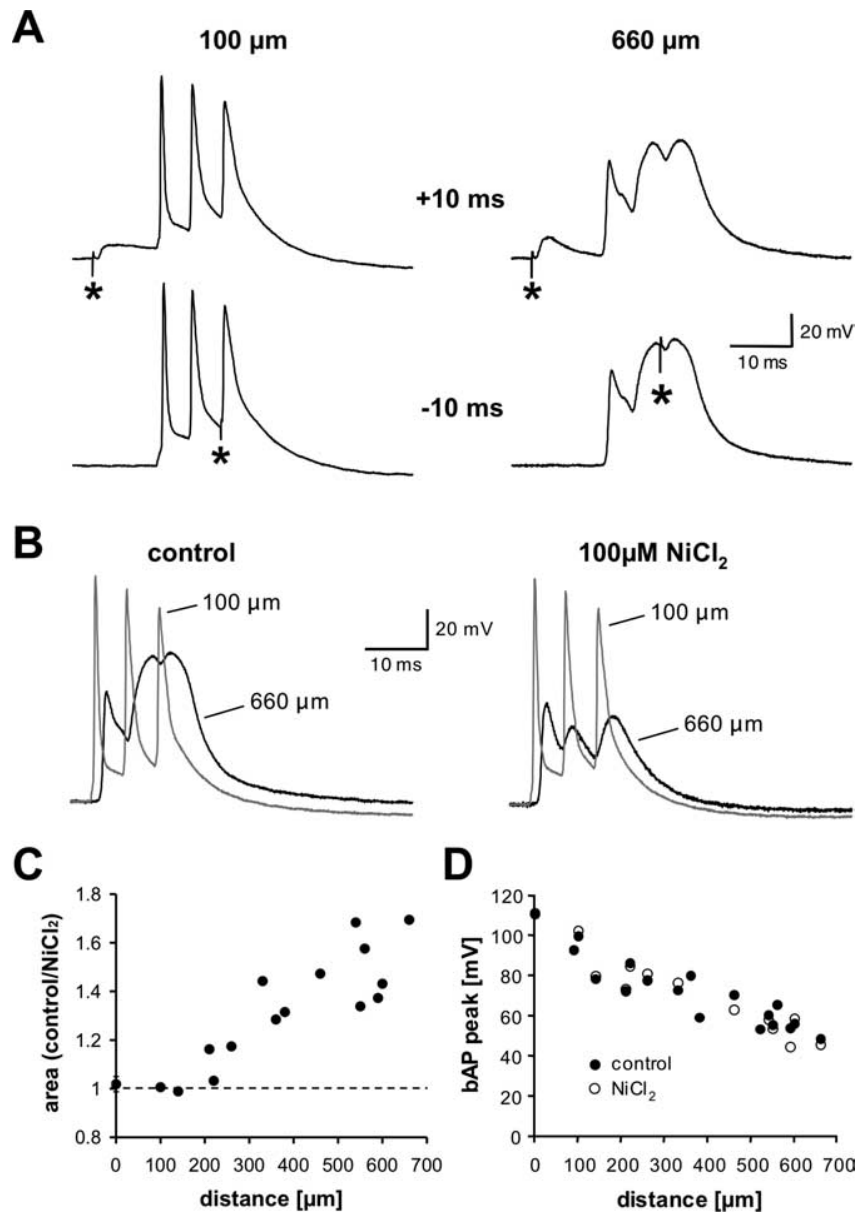

Figure 6. Calcium electrogenesis increases with distance from the soma during AP burst firing. $A$, Local dendritic voltage time course at a proximal ( $100 \mu \mathrm{m}$; left) and a distal ( $660 \mu \mathrm{m}$; right) site during pairing of a somatic AP burst with a locally evoked EPSP at $+10 \mathrm{~ms}$ (top panels) and $-10 \mathrm{~ms}$ (bottom panels). The asterisk marks EPSP onset. $\boldsymbol{B}$, Differential effect of $\mathrm{NiCl}_{2}(100 \mu \mathrm{m})$ on the local voltage waveform during a somatic burst at a proximal (gray; 100 $\mu \mathrm{m}$ ) and distal (black; $660 \mu \mathrm{m}$ ) dendritic site. C, Plot of the distance dependence of the effect of $\mathrm{NiCl}_{2}$ on the voltage integral in response to a somatic burst at various dendritic locations (15 recording sites in 9 neurons). $\boldsymbol{D}$, Absence of an effect of $\mathrm{NiCl}_{2}$ on the amplitude of single backpropagating APs at different dendritic locations (17 recording sites in 9 neurons; $\mathrm{NiCl}_{2}$ applied at 15 sites).

EPSP arrives shortly before the first and largest AP in a burst during pairing at $+10 \mathrm{~ms}$ (Fig. $6 \mathrm{~A}$, top left panel), whereas during pairing at $-10 \mathrm{~ms}$ the EPSP coincides with the last and smallest backpropagating AP (Fig. 6A, bottom left panel). At distal dendritic sites, the situation is reversed, because the first backpropagating $\mathrm{AP}$ is the smallest, and this is followed by dendritic calcium spikes evoked by the second and third APs in the burst (Fig. 6A, right). At these distal locations, therefore, the EPSP arrives at a time significantly before the main dendritic depolarization during pairing at $+10 \mathrm{~ms}$ (Fig. $6 \mathrm{~A}$, top right panel), whereas during pairing at $-10 \mathrm{~ms}$, the EPSP coincides with the peak depolarization in the distal dendrite (Fig. $6 \mathrm{~A}$, bottom right panel).

Blocking T- and/or R-type voltage-gated calcium channels by bath application of $100 \mu \mathrm{M} \mathrm{NiCl}{ }_{2}$ severely reduced the amplitude of dendritic calcium spikes evoked by AP bursts (Fig. 6B), providing direct evidence that dendritic calcium electrogenesis accounts for a large part of the voltage integral during AP bursts at distal dendritic sites. Furthermore, the effect of $\mathrm{NiCl}_{2}$ on the local dendritic voltage integral displayed a steep distance dependence 

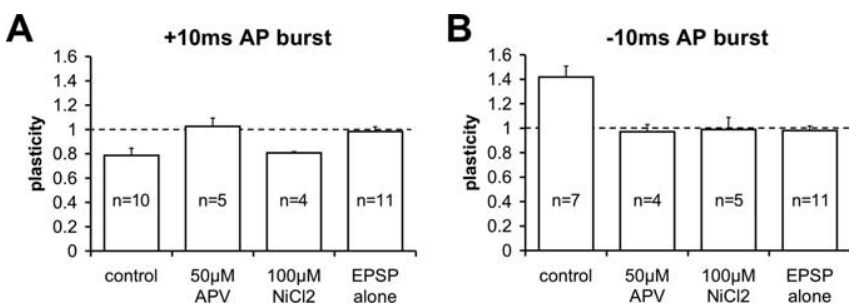

Figure 7. Pharmacology of STDP of distal inputs. All experiments were performed on distal synaptic inputs (uEPSP rise time $>2.7 \mathrm{~ms}$ ). $A$, Pairing of single presynaptic APs with postsynaptic bursts at $+10 \mathrm{~ms}$ causes depression, which is blocked by $50 \mu \mathrm{m} \mathrm{APV}(p<0.05)$, but not affected by $100 \mu \mathrm{M} \mathrm{NiCl} 2(p>0.05)$. uEPSPs elicited without postsynaptic activity $(n=6)$, or with postsynaptic AP bursts offset by $500 \mathrm{~ms}(n=5)$, showed no change in amplitude (EPSP alone; $p>0.05)$. $B$, At $-10 \mathrm{~ms}$ pairing intervals, distal inputs show LTP, which is blocked by both $50 \mu \mathrm{MAPV}(p<0.01)$ and by $100 \mu \mathrm{M} \mathrm{NiCl}(p=0.01)$. Data are presented as mean \pm SEM.

(Fig. 6C), underpinning the notion that dendritic calcium electrogenesis dominates in the distal apical dendrite but has little effect at proximal locations. In contrast, the amplitude of single backpropagating APs was not affected by $\mathrm{NiCl}_{2}$ (Fig. 6D).

\section{Pharmacology of distal synaptic plasticity}

We show here that layer $2 / 3$ synapses impinging onto the distal apical dendrites of layer 5 pyramidal neurons show novel learning rules: when paired with AP bursts they depress during pairing at positive times and potentiate during pairing at negative timings. To understand the mechanisms responsible for this phenomenon, we focused our pharmacological analysis on STDP of distal connections. Application of APV $(50 \mu \mathrm{M})$ abolished LTD at distal synapses after pairing at $+10 \mathrm{~ms}$ with AP bursts (Fig. $7 \mathrm{~A}$ ) $(1.03 \pm 0.07 ; p<0.05)$, indicating a dependence on NMDA receptor activation, whereas $\mathrm{NiCl}_{2}(100 \mu \mathrm{M})$ had no significant effect (Fig. 7A) (0.81 $\pm 0.01 ; p>0.05)$. In contrast, LTP observed at distal synapses after pairing at $-10 \mathrm{~ms}$ with AP bursts was abolished by both APV (Fig. $7 B)(0.97 \pm 0.06 ; p=0.007)$ and $\mathrm{NiCl}_{2}$ (Fig. 7B) $(0.99 \pm 0.10 ; p=0.01)$. Application of $\mathrm{NiCl}_{2}(100$ $\mu \mathrm{M})$ had no significant effect on uEPSP amplitude ( $96 \pm 9 \%$ of control; $p>0.05 ; n=9$ ) or the amplitude of NMDA responses (supplemental Fig. 4, available at www.jneurosci.org as supplemental material).

The most parsimonious interpretation of these findings is that distal LTP requires dendritic calcium electrogenesis mediated by T- or R-type voltage-gated calcium channels, which through sustained dendritic depolarization enhances voltage-dependent unblock of synaptic NMDA receptors. On the other hand, although distal LTD also depends on NMDA receptor activation, and on backpropagating APs as experiments with EPSPs alone show no change, it does not appear to depend on dendritic calcium electrogenesis during AP bursts (Fig. 6A).

\section{Local learning rules change continuously along the apical dendrite of layer 5 pyramidal neurons}

To gain a better understanding of the cellular mechanisms underlying the observed distance dependence of STDP, we modeled STDP induction during postsynaptic AP bursts (Fig. 8). Importantly, these simulations incorporated a recent kinetic model of the voltage-dependent magnesium unblock of the NMDA receptor based on data obtained from layer 5 pyramidal neurons (Kampa et al., 2004). The local voltage time course in these simulations was similar to that observed experimentally during dendritic recordings: at proximal sites, the voltage closely follows the
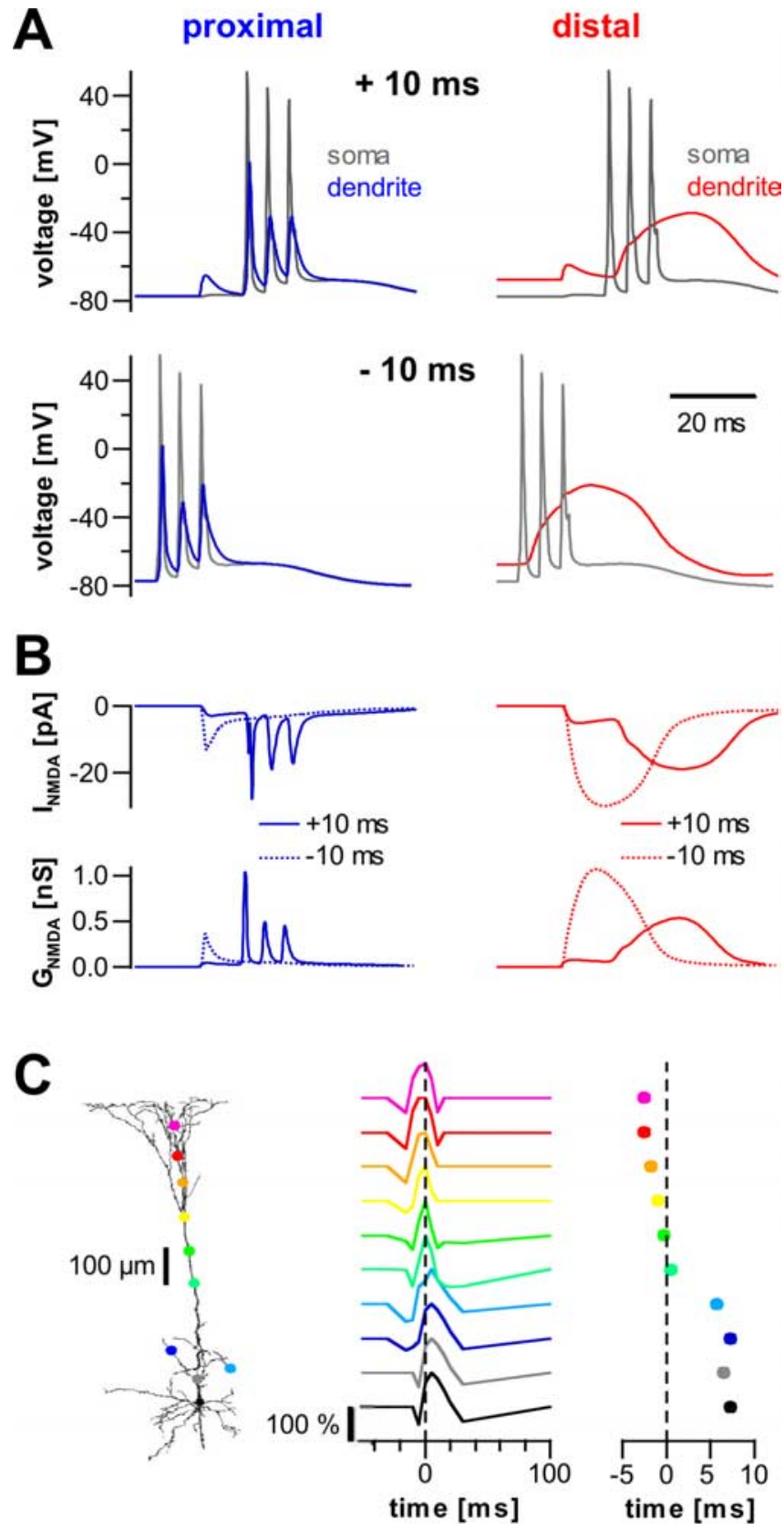

Figure 8. Model of STDP induction in apical dendrites of layer 5 pyramidal neurons. $\boldsymbol{A}$, Membrane voltage at proximal (left; $200 \mu \mathrm{m}$ from soma; blue traces) and distal (right; $800 \mu \mathrm{m}$; red traces) synaptic locations during pairing of AP bursts with synaptic inputs at positive $(+10$ $\mathrm{ms}$; top panels) and negative ( $-10 \mathrm{~ms}$; bottom panels) times in a morphologically realistic model of a layer 5 pyramidal neuron (C). B, NMDA receptor activation (I $I_{\mathrm{NMDA}}$, top; $G_{\mathrm{NMDA}}$ bottom) at proximal and distal locations as in $\boldsymbol{A}$ during pairing at positive (+10 ms) and negative $(-10 \mathrm{~ms})$ times. Note that AP burst-evoked calcium spikes at distal dendritic sites (red) led to greater NMDA receptor activation during pairing at negative compared with positive times. C, Left, Model of the layer 5 pyramidal neuron showing the color coded location of synaptic inputs. The blue (oblique) and red (distal tuft) inputs correspond to the proximal and distal inputs used in $\boldsymbol{A}$ and $\boldsymbol{B}$. Center, Color-coded STDP timing curves for synapses at the dendritic locations in the model. Scale bar indicates $100 \%$ synaptic plasticity. Right, Positive peaks of STDP timing curves (LTP) color-coded for each dendritic site show a shift from positive to negative spike timing with distance from soma.

somatic burst with the peak dendritic depolarization occurring during the first AP (Fig. 8 A, left). Conversely, at distal dendritic sites, maximal depolarization occurs after the third AP in the burst because of generation of dendritic calcium electrogenesis 
(Fig. $8 A$, right). As a consequence, at proximal sites, NMDA receptor activation is maximal at positive timings because the EPSP arrives just before the peak dendritic depolarization, whereas negative timings lead to less NMDA receptor activation because the EPSP coincides with the third and smallest backpropagating AP in the burst (Fig. $8 B$, left). In contrast, at distal sites, the EPSP arrives at the peak depolarization when paired at negative timing intervals, causing maximal NMDA receptor activation, whereas positive timings elicit moderate NMDA receptor activation as the EPSP occurs well before the maximal dendritic depolarization (Fig. $8 B$, right).

The extent of NMDA receptor activation at different locations during pairing with AP bursts was converted into STDP curves based on a threshold model (supplemental Fig. 5, available at www.jneurosci.org as supplemental material) in which strong NMDA receptor activation leads to LTP, moderate NMDA receptor activation leads to LTD, and no plasticity is observed during weak NMDA receptor activation (Shouval et al., 2002). At each synaptic location (Fig. $8 C$, left), we used the integrated NMDA receptor conductance normalized to the maximum observed during pairing. STDP curves calculated for a range of dendritic locations (Fig. $8 C$, center) showed a shift from the canonical timing curve for proximal inputs (LTP at positive, LTD at negative timings), to the novel timing curve described for distal inputs (LTP at negative, LTD at positive timings). This lead to a distance-dependent shift of the best timing for generation of LTP (Fig. 8C, right). These modeling results show that the observed distance dependence of STDP learning rules can be explained by distance-dependent differences in the local voltage waveform driving NMDA receptor activation at different input locations with identical synaptic properties. This suggests that active and passive properties of the apical and oblique dendrites of layer 5 pyramidal neurons are sufficient to explain the experimental observations. Although we cannot rule out a role of potential differences in synaptic properties at proximal and distal synapses, this was not required to explain our findings.

\section{Discussion}

The sign and magnitude of changes in synaptic strength during STDP depend on the relative timing of presynaptic and postsynaptic APs. Although it is commonly accepted that the feedback signal during STDP is mediated by the backpropagating AP, a number of studies indicate that AP backpropagation is decremental in many neuronal types and that backpropagating APs can fail to invade distal dendritic locations. These studies suggest that STDP will depend on synapse location within the dendritic tree. Consistent with this idea, we show here that the learning rules for STDP at synaptic inputs from layer $2 / 3$ to layer 5 pyramidal neurons depend on their dendritic location. This leads to a progressive, distance-dependent shift in the timing requirements for induction of LTP and LTD at proximal and distal synapses during postsynaptic AP bursts. Furthermore, we find that, during AP bursts, distal synapses display novel timing requirements: they potentiate when the UEPSP arrives after the onset of an AP burst (negative timing) and depress when the synapse is activated before burst onset (positive timing). This is in contrast to the timing requirements of proximal synapses, which show LTP at positive and LTD at negative timings, as observed by previous studies (Markram et al., 1997a; Bi and Poo, 1998; Debanne et al., 1998; Feldman, 2000; Sjostrom et al., 2001; Froemke et al., 2005).

\section{Cellular mechanisms}

Most previous studies have shown that induction of STDP depends on activation of NMDA receptors (Magee and Johnston, 1997; Markram et al., 1997a; Bi and Poo, 1998; Debanne et al., 1998; Feldman, 2000; Sjostrom et al., 2001; Froemke et al., 2005). The most parsimonious interpretation of this result is that depolarization associated with backpropagating APs triggers STDP via relief of the voltage-dependent block of the NMDA receptor by magnesium ions (Mayer et al., 1984; Nowak et al., 1984). At positive spike timings, this presumably leads to substantial calcium influx through synaptic NMDA receptors, triggering LTP, whereas at negative timings a moderate NMDA-mediated calcium signal is thought to trigger LTD (Yang et al., 1999; Cho et al., 2001; Cormier et al., 2001; Dan and Poo, 2004).

The pharmacology observed for plasticity of distal layer $2 / 3$ inputs when paired with postsynaptic AP bursts is consistent with this model, because both LTP and LTD were blocked by NMDA receptor antagonists (Fig. 7). Our observations differ from conventional views of STDP, however, in that the timing requirements for STDP depend on synapse location. During pairing at $-10 \mathrm{~ms}$, distal EPSPs coincide with the peak depolarization mediated by dendritic calcium electrogenesis during AP bursts. This would be expected to cause substantial NMDA receptormediated calcium influx, which is likely to underlie the observed LTP. Consistent with this idea, blocking dendritic calcium electrogenesis with $\mathrm{NiCl}_{2}$ abolishes LTP (Fig. 7). Furthermore, simulations indicate that NMDA receptor activation was greatest during pairing at negative times at distal dendritic locations (Fig. 8). In contrast, during pairing at $+10 \mathrm{~ms}$, distal EPSPs occur well before the main dendritic depolarization during AP bursts (Fig. 6), leading to moderate NMDA receptor activation as observed in the model (Fig. 8). This moderate calcium influx is the most likely explanation for the LTD observed at distal synapses at this timing. Application of $\mathrm{NiCl}_{2}$ had no effect on distal LTD (Fig. 7), suggesting that dendritic calcium electrogenesis was not necessary for induction of LTD. In keeping with this interpretation, pairing EPSPs at $+10 \mathrm{~ms}$ with single backpropagating APs, which are associated with minimal calcium electrogenesis, also caused distance-dependent LTD that was greater for more distal inputs (Fig. 2). At proximal apical dendritic locations, calcium electrogenesis contributes little to total dendritic depolarization during AP bursts (Fig. 6C), and backpropagating APs are significantly larger than at distal locations (Fig. 6D). At proximal sites, therefore, it is presumably the APs themselves, rather than any associated dendritic calcium electrogenesis, that trigger both NMDA receptor activation and associated calcium influx. Consistent with this idea, in the model NMDA receptor, activation at proximal locations was greatest during pairing at positive times (Fig. 8). In conclusion, the observed distance dependence of STDP learning rules is most easily explained by distance-dependent differences in local voltage waveforms during single APs and AP bursts, leading to differences in the timing requirements for NMDA receptor activation at proximal and distal sites (Fig. 8).

\section{Comparisons with recent studies}

Two recent studies have examined the potential distance dependence of STDP. Froemke et al. (2005) observed conventional STDP timing requirements for proximal and distal inputs to layer $2 / 3$ pyramidal neurons, but found that the time window for induction of LTD is broader for distal inputs. In addition, Froemke et al. (2005) report that, during pairing at positive times, distal inputs display less LTP than proximal synapses. Sjostrom and Hausser (2006) extended these findings to show that distal inputs 
to layer 5 neurons in fact undergo LTD during pairing at positive times. These results parallel our observation of a distancedependent increase in LTD during pairing of single APs with distal uEPSPs at positive timings (Fig. $2 D$ ). In addition, Sjostrom and Hausser (2006) show that, at distal inputs, dendritic depolarization during STDP induction is able to convert LTD into LTP. This result is complementary to the requirement of AP bursts for LTP induction reported here (Figs. 3, 4), because AP bursts also lead to greater dendritic depolarization than single backpropagating APs (Fig. 6).

\section{Role of AP bursts in STDP}

Pairing single APs at +10 ms elicited LTD (Fig. 2), even when the apical dendrite was depolarized by dendritic current injection subthreshold for BAC-firing during STDP induction (Fig. 3, subthreshold). In contrast, pairing single APs at $+10 \mathrm{~ms}$ with more pronounced dendritic depolarization that led to BAC-firing was sufficient to generate LTP at proximal synapses (Fig. 3, BAC). Similar observations were made during pairing of uEPSPs with AP bursts elicited at the soma (Fig. 4A-D). Conversely, LTP induction at distal synapses was only observed during pairing of AP bursts at negative times $(-10 \mathrm{~ms})$. Previous work indicates that the threshold for initiation of BAC-firing is lowest when the AP precedes the EPSP by 5-10 ms (Larkum et al. 1999a). A possible functional interpretation of the observed LTP at distal inputs during pairing with AP bursts at negative times is therefore that distal inputs are rewarded if they contribute to switching the output mode of the neuron from a single AP to an AP burst.

A requirement for postsynaptic AP burst firing in lowfrequency, associative LTP induction has also been reported in the CA1 region of hippocampus (Pike et al., 1999) and the neocortex (Kampa et al., 2006). Interestingly, Kampa et al. (2006) found that at unitary layer 5 inputs to layer 5 pyramidal neurons pairing AP bursts elicits LTP at both positive and negative times. We also observed LTP induction during pairing at positive and negative times; however, this was found to depend on synapse location, with LTP observed for distal layer $2 / 3$ inputs at negative times and proximal layer $2 / 3$ inputs at positive times. Although not examined in the study by Kampa et al. (2006), a similar dependence of STDP timing rules on synapse location may also exist for layer 5 inputs to layer 5 neurons. This issue is difficult to directly examine at layer 5 to layer 5 synapses because of the relatively short electronic length of basal dendrites, onto which the majority of these inputs are made, and their spatially distributed nature (Markram et al., 1997b). In addition, differences in the timing requirements for STDP at layer 5 to layer 5 inputs compared with layer $2 / 3$ inputs to these neurons may arise because of differences in the active and passive properties of basal versus apical/oblique dendrites, or differences in the synaptic properties of these inputs.

\section{Functional significance of distributed learning rules in layer 5 neurons}

Our results characterize the learning rules governing STDP of layer $2 / 3$ pyramidal inputs onto layer 5 pyramidal neurons. Our finding that the learning rules for STDP depend on synapse location suggests that proximal and distal layer $2 / 3$ inputs convey different information to the postsynaptic layer 5 neuron. At a functional level, a large body of research has highlighted the different impact of distal and proximal synaptic inputs on neuronal output in neurons with extensive dendritic trees (for review, see Williams and Stuart, 2003). In layer 5 pyramidal neurons, distally elicited EPSPs attenuate strongly on their way to the soma, mak- ing them ineffective sources of background excitation (Williams and Stuart, 2002). Conversely, distal synapses are able to generate dendritic calcium spikes through powerful synaptic input (Schiller et al., 1997), coincident distal EPSPs within a brief time window (Williams and Stuart, 2002), or after pairing of distal EPSPs with backpropagating APs at appropriate times (Larkum et al., 1999a). Importantly, the somatic output of this dendritic calcium electrogenesis is typically a burst of APs (Schiller et al., 1997; Larkum et al., 1999a; Williams and Stuart, 1999, 2002). These data suggest that the proximal and distal compartments of layer 5 pyramidal neurons are likely to perform different computational tasks. Our results extend these observations to functional synaptic plasticity, and provide a first description of how inputs may be selected in an activity-dependent manner depending on their location in the dendritic tree.

\section{References}

Bi GQ, Poo MM (1998) Synaptic modifications in cultured hippocampal neurons: dependence on spike timing, synaptic strength, and postsynaptic cell type. J Neurosci 18:10464-10472.

Bliss TV, Lomo T (1973) Long-lasting potentiation of synaptic transmission in the dentate area of the anaesthetized rabbit following stimulation of the perforant path. J Physiol (Lond) 232:331-356.

Cho K, Aggleton JP, Brown MW, Bashir ZI (2001) An experimental test of the role of postsynaptic calcium levels in determining synaptic strength using perirhinal cortex of rat. J Physiol (Lond) 532:459-466.

Connors BW, Gutnick MJ, Prince DA (1982) Electrophysiological properties of neocortical neurons in vitro. J Neurophysiol 48:1302-1320.

Cormier RJ, Greenwood AC, Connor JA (2001) Bidirectional synaptic plasticity correlated with the magnitude of dendritic calcium transients above a threshold. J Neurophysiol 85:399-406.

Dan Y, Poo MM (2004) Spike timing-dependent plasticity of neural circuits. Neuron 44:23-30.

Debanne D, Gahwiler BH, Thompson SM (1998) Long-term synaptic plasticity between pairs of individual CA3 pyramidal cells in rat hippocampal slice cultures. J Physiol (Lond) 507:237-247.

Egger V, Feldmeyer D, Sakmann B (1999) Coincidence detection and changes of synaptic efficacy in spiny stellate neurons in rat barrel cortex. Nat Neurosci 2:1098-1105.

Feldman DE (2000) Timing-based LTP and LTD at vertical inputs to layer II/III pyramidal cells in rat barrel cortex. Neuron 27:45-56.

Froemke RC, Poo MM, Dan Y (2005) Spike-timing-dependent synaptic plasticity depends on dendritic location. Nature 434:221-225.

Gulledge AT, Stuart GJ (2003) Excitatory actions of GABA in the cortex. Neuron 37:299-309.

Hausser M, Roth A (1997) Estimating the time course of the excitatory synaptic conductance in neocortical pyramidal cells using a novel voltage jump method. J Neurosci 17:7606-7625.

Hines ML, Carnevale NT (1997) The NEURON simulation environment. Neural Comput 9:1179-1209.

Jack JJ, Miller S, Porter R, Redman SJ (1971) The time course of minimal excitory post-synaptic potentials evoked in spinal motoneurones by group Ia afferent fibres. J Physiol (Lond) 215:353-380.

Kampa BM, Clements J, Jonas P, Stuart GJ (2004) Kinetics of $\mathrm{Mg}^{2+}$ unblock of NMDA receptors: implications for spike-timing dependent synaptic plasticity. J Physiol (Lond) 556:337-345.

Kampa BM, Letzkus JJ, Stuart GJ (2006) Requirement of dendritic calcium spikes for induction of spike-timing dependent synaptic plasticity. J Physiol (Lond) 574:283-290.

Kole MH, Hallermann S, Stuart GJ (2006) Single $I_{\mathrm{h}}$ channels in pyramidal neuron dendrites: properties, distribution, and impact on action potential output. J Neurosci 26:1677-1687.

Larkum ME, Zhu JJ, Sakmann B (1999a) A new cellular mechanism for coupling inputs arriving at different cortical layers. Nature 398:338-341.

Larkum ME, Kaiser KM, Sakmann B (1999b) Calcium electrogenesis in distal apical dendrites of layer 5 pyramidal cells at a critical frequency of back-propagating action potentials. Proc Natl Acad Sci USA 96:14600-14604.

Larkum ME, Zhu JJ, Sakmann B (2001) Dendritic mechanisms underlying the coupling of the dendritic with the axonal action potential initiation 
zone of adult rat layer 5 pyramidal neurons. J Physiol (Lond) 533:447-466.

Llinas RR (1988) The intrinsic electrophysiological properties of mammalian neurons: insights into central nervous system function. Science 242:1654-1664

Lynch GS, Dunwiddie T, Gribkoff V (1977) Heterosynaptic depression: a postsynaptic correlate of long-term potentiation. Nature 266:737-739.

Magee JC, Johnston D (1997) A synaptically controlled, associative signal for Hebbian plasticity in hippocampal neurons. Science 275:209-213.

Manns ID, Sakmann B, Brecht M (2004) Sub- and suprathreshold receptive field properties of pyramidal neurones in layers $5 \mathrm{~A}$ and $5 \mathrm{~B}$ of rat somatosensory barrel cortex. J Physiol (Lond) 556:601-622.

Markram H, Lubke J, Frotscher M, Sakmann B (1997a) Regulation of synaptic efficacy by coincidence of postsynaptic APs and EPSPs. Science 275:213-215.

Markram H, Lubke J, Frotscher M, Roth A, Sakmann B (1997b) Physiology and anatomy of synaptic connections between thick tufted pyramidal neurones in the developing rat neocortex. J Physiol (Lond) 500:409-440.

Martin SJ, Grimwood PD, Morris RG (2000) Synaptic plasticity and memory: an evaluation of the hypothesis. Annu Rev Neurosci 23:649-711.

Mayer ML, Westbrook GL, Guthrie PB (1984) Voltage-dependent block by $\mathrm{Mg}^{2+}$ of NMDA responses in spinal cord neurones. Nature 309:261-263.

Nowak L, Bregestovski P, Ascher P, Herbet A, Prochiantz A (1984) Magnesium gates glutamate-activated channels in mouse central neurones. $\mathrm{Na}$ ture 307:462-465.

Pike FG, Meredith RM, Olding AW, Paulsen O (1999) Rapid report: postsynaptic bursting is essential for "Hebbian" induction of associative long-term potentiation at excitatory synapses in rat hippocampus. J Physiol (Lond) 518:571-576.

Reyes A, Sakmann B (1999) Developmental switch in the short-term modification of unitary EPSPs evoked in layer 2/3 and layer 5 pyramidal neurons of rat neocortex. J Neurosci 19:3827-3835.

Schaefer AT, Larkum ME, Sakmann B, Roth A (2003) Coincidence detection in pyramidal neurons is tuned by their dendritic branching pattern. J Neurophysiol 89:3143-3154.

Schiller J, Schiller Y, Stuart G, Sakmann B (1997) Calcium action potentials restricted to distal apical dendrites of rat neocortical pyramidal neurons. J Physiol (Lond) 505:605-616.
Shouval HZ, Bear MF, Cooper LN (2002) A unified model of NMDA receptor-dependent bidirectional synaptic plasticity. Proc Natl Acad Sci USA 99:10831-10836.

Sjostrom PJ, Hausser M (2006) A cooperative switch determines the sign of synaptic plasticity in distal dendrites of neocortical pyramidal neurons. Neuron 51:227-238.

Sjostrom PJ, Turrigiano GG, Nelson SB (2001) Rate, timing, and cooperativity jointly determine cortical synaptic plasticity. Neuron 32:1149-1164.

Spruston N, Schiller Y, Stuart G, Sakmann B (1995) Activity-dependent action potential invasion and calcium influx into hippocampal CA1 dendrites. Science 268:297-300.

Stuart G, Spruston N (1998) Determinants of voltage attenuation in neocortical pyramidal neuron dendrites. J Neurosci 18:3501-3510.

Stuart GJ, Hausser M (2001) Dendritic coincidence detection of EPSPs and action potentials. Nat Neurosci 4:63-71.

Stuart GJ, Sakmann B (1994) Active propagation of somatic action potentials into neocortical pyramidal cell dendrites. Nature 367:69-72.

Waters J, Helmchen F (2004) Boosting of action potential backpropagation by neocortical network activity in vivo. J Neurosci 24:11127-11136.

Watt AJ, Sjostrom PJ, Hausser M, Nelson SB, Turrigiano GG (2004) A proportional but slower NMDA potentiation follows AMPA potentiation in LTP. Nat Neurosci 7:518-524.

Williams SR, Stuart GJ (1999) Mechanisms and consequences of action potential burst firing in rat neocortical pyramidal neurons. J Physiol (Lond) 521:467-482.

Williams SR, Stuart GJ (2000a) Site independence of EPSP time course is mediated by dendritic $I_{\mathrm{h}}$ in neocortical pyramidal neurons. J Neurophysiol 83:3177-3182.

Williams SR, Stuart GJ (2000b) Backpropagation of physiological spike trains in neocortical pyramidal neurons: implications for temporal coding in dendrites. J Neurosci 20:8238-8246.

Williams SR, Stuart GJ (2002) Dependence of EPSP efficacy on synapse location in neocortical pyramidal neurons. Science 295:1907-1910.

Williams SR, Stuart GJ (2003) Role of dendritic synapse location in the control of action potential output. Trends Neurosci 26:147-154.

Yang SN, Tang YG, Zucker RS (1999) Selective induction of LTP and LTD by postsynaptic $\left[\mathrm{Ca}^{2+}\right]_{\mathrm{i}}$ elevation. J Neurophysiol 81:781-787. 